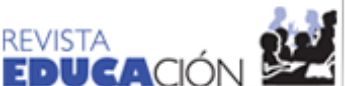

Revista Educación

ISSN: 0379-7082

ISSN: 2215-2644

revedu@gmail.com

Universidad de Costa Rica

Costa Rica

\section{Transferencia de la formación en competencias de liderazgo escolar en una universidad chilena}

Parra Robledo, Richar; Ruiz Bueno, Carmen

Transferencia de la formación en competencias de liderazgo escolar en una universidad chilena

Revista Educación, vol. 44, núm. 2, 2020

Universidad de Costa Rica, Costa Rica

Disponible en: http://www.redalyc.org/articulo.oa?id=44062184023

DOI: https://doi.org/10.15517/revedu.v44i2.38945

Esta obra está bajo una Licencia Creative Commons Atribución-NoComercial-SinDerivar 3.0 Internacional. 


\section{Transferencia de la formación en competencias de liderazgo escolar en una universidad chilena}

\section{Transference of School Administrative Leadership Skills Training at a University in Chile}

Richar Parra Robledo

Universidad Autónoma de Barcelona, España

richar.parra.robledo@gmail.com

iD http://orcid.org/0000-0002-8988-5807

Carmen Ruiz Bueno

Universitat Autònoma de Barcelona, España

carmen.ruiz.bueno@uab.cat

(iD) http://orcid.org/0000-0003-3694-3150
DOI: https://doi.org/10.15517/revedu.v44i2.38945

Redalyc: http://www.redalyc.org/articulo.oa?id=44062184023

Recepción: 12 Febrero 2020

Aprobación: 04 Junio 2020

\section{Resumen:}

El presente artículo tiene como objetivo evaluar la transferencia de la formación en competencias de liderazgo directivo escolar en una universidad chilena, en el marco del Plan de formación de directoras y directores en Chile. El tipo de investigación es no experimental, transaccional descriptiva. Para recoger la información, se diseñó un cuestionario de transferencia, el cual posee un índice elevado de consistencia interna global $(\alpha=0,920)$. El análisis de los resultados se establece en base a cuatro categorías de factores: 1) personales, 2) de la formación, 3) de la organización y 4) transferencia de competencias. Convergiendo en el diseño de un esquema de los factores facilitadores y obstaculizadores de la transferencia de competencias de liderazgo directivo escolar. Sumado a lo anterior, el estudio desarrolla y evalúa el constructo de transferibilidad de las competencias de la formación como la capacidad de resignificar y recontextualizar las competencias en diferentes contextos, desde la perspectiva de la movilidad profesional. Las conclusiones se focalizan en tres aspectos claves: 1) a mayor motivación por aprender mayor desarrollo personal, 2) el diseño curricular y la coherencia interna de la formación ofrece mayores posibilidades de transferencia y 3) al aumentar la seguridad profesional y fomentar el trabajo en equipo se fortalece el liderazgo pedagógico.

Palabras Clave: Transferencia, Formación, Evaluación, Competencias, Liderazgo.

\section{Abstract:}

The objective of this article is to assess information transfer for educational administration leadership skills programs at a Chilean university within the framework of Chile's School Principal Leadership Program. The research carried out for this study is nonexperimental, transactional and descriptive. The process for gathering data involved a data transfer questionnaire which includes a high global internal consistency index $(\alpha=0,920)$. Analysis of the results was based on four aspects: 1$)$ personal factors, 2 ) educational factors, 3 ) organizational factors and 4) skills transfer. These are combined to design an outline focusing on aspects that facilitate and hinder the transfer of school administration leadership skills training. This study also develops and assesses how skills provide opportunities for reconsidering and recontextualizing associated contexts. The conclusions that were drawn focus on three key aspects: 1) Greater motivation to learn and further one's personal development, 2) Curricular design and internal continuity and coordination in developing skills training possibilities and promote skills transfer. 3) Enhanced job security and encouraging team work strengthens pedagogical leadership.

KEYWORDS: Transfer, Training, Assessment, Skills, Leadership.

\section{INTRODUCCIÓN}

La evaluación de la formación se define como una serie de procesos de recogida de información orientado a la emisión de juicios de mérito o valor respecto a una acción formativa (Cano, 2016). Subyace de la definición 
anterior, el concepto de toma de decisiones, las cuales deben encaminarse a las mejoras internas y externas de las acciones formativas. En sí, la evaluación proporciona información importante para identificar y resolver las debilidades de la acción formativa, favoreciendo los bucles de mejora. Por tanto, para un proceso sistemático y planificado de evaluación, se debe considerar las diferentes etapas del proceso, y a la vez, responder a las diferentes etapas de las acciones formativas.

Una de las etapas de la formación, es la puesta en práctica de lo aprendido en el puesto de trabajo, la transferencia. Se define transferencia como el grado en que se aplican efectivamente los conocimientos, habilidades y actitudes, adquiridas en las acciones de la formación, en el contexto del trabajo (Baldwin y Ford, 1988; González, Alonso y Berrocal, 2017; López, Fijoo, Novel y Leyva, 2017).

El estudio tiene como objetivo evaluar la transferencia de la formación en competencias de liderazgo directivo escolar en una universidad en Chile. Por tanto, y considerando el amplio espectro, se decidió desarrollar un doble marco teórico, el primero, vinculado a la transferencia de la formación y el segundo, circunscrito a la formación de líderes y lideresas escolares.

La investigación cobra importancia investigativa dadas las particularidades y características del programa formativo, el cual se inserta en una política educativa del ámbito escolar en Chile. Por cuando es relevante generar una reflexión desde los aprendizajes de las personas participantes sobre las condiciones e implicaciones de la acción formativa en su contexto (Bernal, 2006).

\section{La transferencia de la formación}

La transferencia de la formación al puesto de trabajo, es el desarrollo efectivo y práctico del aprendizaje en el ejercicio del cargo, en sí, es la aplicación en la práctica. En específico, es el cambio de comportamiento efectivo en el puesto de trabajo, incluyendo aspectos como el saber ser y saber hacer, y el clima laboral (González et al., 2017; Kirkpatrick, 2000; López et al., 2017; Rent, 2013).

El estudio se posiciona desde la perspectiva de dos referentes de la evaluación de la transferencia, Baldwin y Ford (1988) y Holton (1996), por cuanto, las personas autoras vinculan una serie de factores y variables con la transferencia de los aprendizajes al puesto de trabajo. Los factores de la transferencia hacen referencia a los aspectos personales de las personas participantes y aspectos externos, como factores interpersonales, de la formación, contextuales y organizaciones que pueden facilitar u obstaculizar la aplicación de lo aprendido en la organización (Baldwin y Ford, 1988; Cejas y Navío, 2017; Holton, 1996; Kirkpatrick, 2000).

En la presente investigación, se incorpora el concepto de transferibilidad de las competencias en la formación, la cual resitúa la transferencia, a la capacidad de resignificar y recontextualizar las competencias, y a su vez, aplicarlas a otros contextos, considerando la movilidad profesional. La transferibilidad aplicada a la formación nace a la luz de la unificación de créditos y titulaciones en el espacio de la Unión Europea (Costales, Rosales y Acosta, 2016). Respecto a la fuerte relación con el aprendizaje de competencias y la capacidad de resignificar, lo cual moviliza las competencias a las diferentes y cambiantes situaciones de la práctica.

La investigación de Romero, Rodríguez y Gutiérrez (1999), platean que la transferibilidad está íntimamente conectada con el aprendizaje de competencia y su relación entre formación y la organización. La Oficina Internacional del Trabajo (OIT), se refiere a la transferibilidad, en términos de la calificación transferible y como la capacidad de uso productivo en distintos contextos, trabajos y organizaciones (Organización Internacional del Trabajo [OIT], 2007).

El estudio de Costales et al. (2016) posiciona la transferibilidad desde la perspectiva de la OIT, aportando nuevas líneas desde diversidad de empleos y movilidad profesional. Por tal motivo, se define operacionalmente la transferibilidad como la capacidad de transferir la formación a otros contextos por parte de las personas participantes (Costales et al., 2016; OIT, 2007). 


\section{La formación de directoras y directores escolares}

Las políticas educativas internacionales están fortaleciendo el liderazgo escolar, fundamentados en investigaciones nacionales e internacionales, las cuales evidencian la contribución en el logro del aprendizaje, siendo considerado el segundo factor interno de mejora, después de la acción docente (González, Silva y Sepúlveda, 2016; Leithwood, Harris y Hopkins, 2008; Ministerio de Educación de Chile [MINEDUC], 2016, Oficina Regional de Educación para América Latina y el Caribe [OREALC/UNESCO], 2016; Robinson, Hohepa y Lloyd, 2009).

Los hallazgos colegidos por los estudios teóricos y empíricos decantan en la influencia del líder o lideresa en su equipo de trabajo, la generación de un propósito compartido y una articulación de las metas, sumado a los consensos necesarios para el desarrollo del cambio para la mejora escolar. Por cuanto, el equipo directivo motivará al personal docente para mejorar la calidad de sus prácticas pedagógicas, promoviendo estructuras para la colaboración efectiva en la comunidad escolar (Cebrián, 2018; García y Caballero, 2019)

Considerando la relevancia del tema, la Organización para la Cooperación y el Desarrollo Económicos (OCDE) ha desarrollado una serie de recomendaciones para fortalecer el liderazgo educativo, mediante políticas específicas (Pont, Nusche y Moorman, 2008). Las propuestas de abordaje de las sugerencias entregadas por la OCDE, han sido transferidas en una heterogeneidad de experiencias entre los países, traduciéndose en diferentes estructuras de soporte, tanto en la mejora en los perfiles directivos, la revisión de funciones del cargo, diseño de una carrera directiva, certificación nacional, investigaciones en el área y programas de formación directiva con claras directrices (Cancino y Vera, 2017; Cebrián, 2018; Centro de desarrollo del liderazgo educativo [CEDLE], 2018; González et al., 2016).

Respecto a los hallazgos anteriores, se puede señalar la importancia de un equipo directivo profesional, especializado y preparado para la dirección escolar. Al identificar las diferentes propuestas de fortalecimiento del liderazgo escolar, la presente investigación se focaliza en la formación directiva en las cuales se desarrollen competencias y habilidades para una gestión y liderazgo escolar efectivo.

En este sentido cobra importancia determinar las competencias y habilidades claves para el fortalecimiento de un liderazgo escolar efectivo. Los hallazgos y las conclusiones de los estudios internacionales, convergen en cuatro competencias del liderazgo directivo escolar, centradas en prácticas efectivas, las cuales son: (1) visión estratégica, (2) desarrollo del personal, (3) rediseño de la organización y (4) gestión de la enseñanzaaprendizaje (Leithwood, Day, Sammons, Harris y Hopkins, 2006; Pont et al., 2008).

En referencia a Chile, la formación en temas de gestión y liderazgo directivo escolar estuvo marcada por una política reactiva por parte del Ministerio de Educación (MINEDUC), sumado al poco impacto del personal directivo en la organización escolar. Sumado a la falta de alineación interna, la falta de atención a las características de las personas participantes, presentando una fuerte homogeneidad en la oferta académica y una limitada contextualización a la realidad local y nacional (Muñoz y Marfán, 2011). En el 2010, el MINEDUC efectuó un estudio sobre la formación de personas que ejercen la dirección, convergiendo en las conclusiones de las investigaciones anteriores (MINEDUC, 2016).

Dado el contexto y la contundencia de los estudios nacionales e internacionales, el MINEDUC y Centro de Perfeccionamiento, Experimentación e Investigación Pedagógica (CPEIP), dependiente del anterior, desarrollaron un Plan de formación de directoras y directores (PFD) (2011), inserto en la Política educativa de fortalecimiento del liderazgo directivo escolar desde el 2014, involucrando una serie de directrices, las cuales, deben entregar un soporte a la política y generar un impacto en los resultados de aprendizaje del estudiantado (MINEDUC, 2016).

El posicionamiento desde el MINEDUC, se enfoca en transformar la gestión escolar desde una perspectiva administrativa y burocrática de la función directiva en un liderazgo centrado en lo pedagógico desarrollado por el personal directivo. Para tales fines, implementó un Plan nacional de formación de directoras y directores (MINEDUC, 2011). Los lineamientos del Plan de formación son desarrollar competencias y 
prácticas asociadas a la acción directiva y el liderazgo escolar, en cuatro dimensiones de aprendizaje específicas: (1) establecer una dirección clara; (2) desarrollar a las personas, (3) rediseñar la organización escolar y (4) gestionar el programa de enseñanza (MINEDUC, 2011). Dichas prácticas de liderazgo escolar, se concretan en propuestas de acciones formativas, diseñadas por instituciones de educación superior (IES), las cuales concursan de forma pública para adjudicarse los fondos de implementación.

En el ciclo de desarrollo del PFD, según datos públicos de las cohortes 2011-2016, se han ejecutado 104 acciones formativas, favoreciendo a 4.277 participantes a nivel nacional (Centro de Perfeccionamiento, Experimentación e Investigación Pedagógica [CPEIP], 2018). En 2014, se presentaron 33 IES, con una convocatoria total de 45 programas en liderazgo escolar. El proceso de evaluación para la constitución de las acciones formativas del PFD seleccionó 19 IES y 26 propuestas (MINEDUC, 2014). La propuesta formativa, magíster en gestión directiva (Universidad Mayor, 2014), presentada por la escuela de Educación de la Universidad Mayor (Temuco, Chile) en alianza con Universidad de Arizona (Estado Unidos) y la Universidad de Sevilla (España), fue una de las seleccionadas y es la fuente del presente estudio.

En la formación participaron 35 personas, los detalles de la composición del grupo de participantes fue: género; femenino (69\%) - masculino (31\%); cargo al momento de postular; docente y personal de coordinación académica (52\%), equipos directivos (37\%) y profesionales sin cargo directivo (11\%).

En el transcurso de la ejecución del PFD (2011-2020), se han desarrollado distintas evaluaciones y reformulaciones internas. En 2013, el Centro de Estudios del MINEDUC (CEM) midió los avances de las personas participantes, por medio de un cuestionario auto administrado, el cual fue calificado con bajos niveles de fiabilidad (Centro de Estudios del Ministerio de Educación [CEM], 2013). En el 2014, el Plan se reformula en una propuesta más amplia, sistemática y estructura, llamada Política de fortalecimiento del liderazgo escolar, desde el mismo año, se inician las evaluaciones ex ante por parte del Ministerio de Desarrollo Social.

En la actualidad, las investigaciones de evaluación del Plan de formación de directoras y directores escolares son escasas y circunscritas a validaciones internas ex ante. Por tanto, es imprescindible aumentar los vacíos en la literatura y a la vez, incrementar los estudios sobre las transferencia e impacto de la formación en el grupo de participantes, en las organizaciones escolares y en la sociedad.

Por tanto, es importante relevar a un primer lugar la perspectiva de las personas participantes de la formación, con el propósito de desarrollar bucles de mejora de las acciones formativas. Además, cobra preminencia la evaluación como una fuente de transparencia de la gestión pública del Estado y la rendición de cuenta ante la sociedad civil.

El propósito del estudio es evaluar la transferencia del magíster en gestión directiva de la universidad Mayor, en el marco del Plan de formación de directoras y directores, en su cohorte 2014-2015. El programa de formación, magíster en gestión directiva, es un caso particular y específico, desarrollado en una población reducida, con singularidades de las personas participantes, por tanto, es relevante por las particularidades y complejidades de la experiencia formativa (Aneas, Sánchez y Sánchez, 2018; Bernal, 2006).

\section{Metodología}

La investigación se desarrolló bajo el alero del diseño no experimental, ex post facto (Hernández, Fernández y Baptista, 2010), con un muestro intencionado de 26 personas participantes de la cohorte 2014-2015 del máster en gestión directiva de la Universidad Mayor en Temuco, Chile. La justificación de la elección de la metodología cuantitativa no experimental, esta contextualizada por la observación de las condiciones estructurales del programa formativo y las variables sin intervención del investigador.

El estudio de caso se ejecuta considerando las singularidades de la formación directiva, atendiendo a lo específico y complejo del funcionamiento curricular del desarrollo de competencias directivas y de liderazgo escolar. En cuanto al tamaño de la muestra, es importante relevar lo significativo de la experiencia de 
formación para el ejercicio de directores y directoras, y de esta forma lograr una mayor comprensión y profundización de las interrelaciones formativas en situaciones reales en su contexto (Bernal, 2006).

En este aspecto, cobra especial importancia, reconocer las particularidades de la acción formativa seleccionada, en cuanto, es un magíster de formación directiva para profesionales de la educación, con el propósito de desarrollar líderes y lideresas escolar con competencias directivas actualizadas y capaces de generar la transformación en la gestión y el liderazgo directivo escolar en cada centro escolar y de esta forma mejorar los resultados académicos.

Procedimiento. En el primero paso, se diseñó una matriz de sistematización de la revisión bibliográfica, la cual sintetizaba los diferentes referencias, los constructos, dimensiones, reactivos y enfoques de los estudios ejecutados en el área de la transferencia de la formación y de la instrucción de personal directivos escolares. Desde la construcción de la matriz, se desarrolló un posicionamiento teórico/práctico de la evaluación de la transferencia, sustentados en Baldwin y Ford (1988) y Holton (1996), por cuanto, los autores identifican una serie de factores asociados a la transferencia de los aprendizajes a la organización escolar. Posterior al proceso de posicionamiento, se diseñó un cuestionario para evaluar la transferencia de la formación en competencias de liderazgo directivo escolar. Finalizado el procedimiento, se solicitó la validación del instrumento por 14 personas expertas en temas de detección de necesidades formativas y en la formación de personal directivo escolar, con el objetivo de revisar su contenido y validez interna. Los criterios de selección de las personas expertas fueron: (1) publicaciones en revista de divulgación académica, (2) proyectos de investigación en el campo y (3) presentación de comunicaciones del tema en seminarios o congresos (Aneas et al., 2018; Escobar y Cuervo, 2008).

El juicio de personas expertas es una opinión informada de profesionales con trayectoria en el área de estudio, los cuales se encuentran cualificados profesional y académicamente. Los criterios de examinación de las personas expertas fueron:

- Univocidad: nivel de precisión lingüística de la formulación del ítem.

- Pertinencia: adecuación, idoneidad y/o relación del ítem con el objeto de estudio.

- Importancia: peso específico del ítem al conjunto del cuestionario.

Concluida la etapa de la validación, se procedió a ejecutar ajustes gramaticales, solicitados por las personas expertas. Los ajustes en los ítems se operacionalizaron en una secuencia lógica, ágil y amigable, para mejorar la comprensión de los participantes. El cuestionario final presenta cuatro dimensiones con 27 ítems, con un índice elevado de consistencia interna global $(\alpha=0,920)$, como se observa en la Tabla 1.

TABLA 1

Dimensiones del cuestionario

\begin{tabular}{llll}
\hline Dimensión & Descripción & $\mathrm{N}^{\mathbf{0}}$ ítems & $\alpha$ estandarizado \\
\hline Factores personales & $\begin{array}{l}\text { La dimensión se circunscribe a los factores } \\
\text { individuales vinculados a las percepciones del } \\
\text { participante, en temas focalizados a su desarrollo } \\
\text { personal. }\end{array}$ & 5 & 0,902 \\
\hline Factores de la formación & $\begin{array}{l}\text { La dimensión se enfocan en los factores de la } \\
\text { estructura del plan de estudios de la formación, } \\
\text { considerando el diseño curricular. }\end{array}$ & 6 & 0,940 \\
\hline Factores de la organización & $\begin{array}{l}\text { La dimensión considera factores de la organización } \\
\text { vinculados a la aplicación de la formación y el } \\
\text { desarrollo competencial, en el puesto de trabajo. }\end{array}$ & 6 & 0,916 \\
\hline Transferencia de competencias & $\begin{array}{l}\text { En la dimensión se valoran las percepción de los } \\
\text { participantes en torno al desarrollo de las } \\
\text { competencias de liderazgo directivo escolar pos } \\
\text { formación y a su vez, la transferibilidad de la } \\
\text { instrucción y las competencias. }\end{array}$ & 10 & 0,936 \\
& & & \\
\hline
\end{tabular}


En el quinto paso, se envió el cuestionario a los correos electrónicos de las personas participantes por medio de Google forms. Terminado el proceso de recogida de datos, se codificaron los resultados para el análisis en el programa SPSS Statistics 22. El cuestionario final es descrito en la Tabla 2:

TABLA 2

Dimensiones e ítems del cuestionario

\begin{tabular}{|c|c|}
\hline Dimensión & Ítems \\
\hline \multirow{7}{*}{ Factores personales } & a. Expectativas de la formación \\
\hline & 1. Actualizar conocimientos personales \\
\hline & 2. Interés en los temas de gestión directiva \\
\hline & 3. Motivación por aprender. \\
\hline & b. Contribución de la formación \\
\hline & 4. A su desarrollo personal. \\
\hline & 5. Al desarrollo de competencias. \\
\hline \multirow{8}{*}{ Factores de la formación } & a. Diseño curricular \\
\hline & 6. Los objetivos respondieron a sus necesidades. \\
\hline & 7. Los contenidos promovieron la reflexión pedagógica. \\
\hline & b. Estrategias metodológicas \\
\hline & 8. El personal docente favoreció un clima de aprendizaje. \\
\hline & 9. El programa promovió el trabajo cooperativo entre las personas participantes. \\
\hline & 10. El personal docente analizó los nudos de aprendizaje de los contenidos no alcanzados. \\
\hline & $\begin{array}{l}\text { 11. El personal docente utilizó feedforward (sugerencias que ayudan a realizar cambios positivos en su } \\
\text { futuro) en su proceso formativo. }\end{array}$ \\
\hline \multirow{5}{*}{ Factores de la organización } & a. Apoyo de la organización \\
\hline & 12. El clima de la organización facilita la aplicabilidad de las competencias adquiridas en la formación. \\
\hline & $\begin{array}{l}\text { 13. La formación recibida contribuye a la mejora del clima de trabajo. } \\
\text { 14. Existe una cultura de aprendizaje continuo en la organización. }\end{array}$ \\
\hline & 15. La organización permite procesos de innovación pedagógica. \\
\hline & $\begin{array}{l}\text { b. Apoyo de equipos } \\
\text { 16. El equipo directivo de la organización apoya los procesos de transferencia. } \\
\text { 17. El equipo de profesionales de la organización contribuyó a los procesos de transferencia. }\end{array}$ \\
\hline \multirow{7}{*}{ Transferencia de competencias } & $\begin{array}{l}\text { a. Desarrollo de competencias } \\
\text { 18. Desarrollo de seguridad profesional } \\
\text { 19. Liderazgo pedagógico } \\
\text { 20. Gestión directiva } \\
\text { 21. Trabajo en equipo } \\
\text { 22. Resolución de conflicto }\end{array}$ \\
\hline & b. Transferibilidad de la formación \\
\hline & 23. La formación recibida es aplicable a todo contexto escolar. \\
\hline & $\begin{array}{l}\text { 24. Las competencias recibidas en la formación sobre liderazgo pedagógico son aplicables a todo contexto } \\
\text { educativo. }\end{array}$ \\
\hline & $\begin{array}{l}\text { 25. Las competencias recibidas en la formación sobre gestión directiva son aplicables a todo contexto } \\
\text { educativo. }\end{array}$ \\
\hline & $\begin{array}{l}\text { 26. Las competencias recibidas en la formación sobre gestión curricular son aplicables a todo contexto } \\
\text { educativo. }\end{array}$ \\
\hline & $\begin{array}{l}\text { 27. Las competencias recibidas en la formación sobre resolución de conflicto son aplicables a todo contexto } \\
\text { educativo. }\end{array}$ \\
\hline
\end{tabular}

Fuente: Elaboración propia.

\section{ANÁLISIS DE LOS RESULTADOS}

El análisis de los resultados se ejecutó en cuatro apartados: 1) caracterización de la muestra, 2) resultados descriptivos, 3) factores facilitadores y obstaculizadores de la transferencia y 4) correlaciones entre dimensiones.

\section{1) Caracterización de la muestra}

- Género, existe una distribución desigual, 65\% femenino y un 35\% de masculino.

- Edad, la edad media es de 39 años, en un intervalo entre 30 años y 52 años.

- Cargo actual, un 52\% de las personas participantes en el estudio, son docentes y personal de la coordinación académica, en segundo lugar, se encuentra el personal directivo con un 37\%. 
- Dependencia en que trabajan, está focalizada en centros escolares del sector público (69\%) seguido por un $23 \%$ de centros educativos subvencionados o concertados.

\section{2) Resultados descriptivos}

El siguiente apartado se elabora en base a las valoraciones de las percepciones de las personas participantes, las dimensiones de análisis de los resultados son: a) factores personales, b) factores de la formación, c) factores de la organización y d) transferencia de competencias.

Sumado a la revisión de las valoraciones, se ha decidido generar un criterio de categorización, considerando las puntuaciones en una escala de valoración de 1 a 5, estimando aquellos factores sobre la media 3 como factores fuertes y aquellos con menor valoración como factores débiles de transferencia.

a) Factores personales. La dimensión de factores personales se vinculada a las percepciones de las personas participantes, en temas focalizados a su desarrollo personal. Al consultar sobre sus expectativas al momento de inscribirse en el magíster, las puntuaciones se posicionan en el interés por los temas de gestión directiva $(4,84)$, la motivación por aprender $(4,69)$ y la actualización de conocimientos $(4,65)$. En torno a la contribución formación, el grupo de participantes valoran positivamente la acción formativa por su aportación al desarrollo personal $(4,15)$ y al desarrollo de competencias $(4,15)$. Todas las variables mencionadas son factores fuertes de la transferencia.

b) Factores de la formación. La dimensión considera el diseño curricular desde el punto de vista de la estructura del plan de estudios. Entre los factores fuerte de transferencia, se aprecian en la promoción de la reflexión pedagógica en los contenidos tratados $(4,15)$ y la consecución de objetivos de la formación $(3,92)$. Los factores con mayor valoración en el subnivel de las estrategias metodológicas, son la promoción del trabajo cooperativo $(4,26)$ y el personal docente propició un clima favorable para el aprendizaje $(3,84)$. Los factores con menor puntuación, se vinculan a la falta de análisis de los nudos de aprendizaje no logrados $(2,69)$ y la limitada utilización de feedforward por parte del equipo docente durante el proceso formativo $(2,96)$. Ambos son factores débiles de transferencia.

c) Factores de la organización. La dimensión identifica los factores vinculados a la aplicación de la formación y el desarrollo competencial en el puesto de trabajo. Los factores fuertes en la organización están asociadas al clima laboral, concretamente en la contribución de formación en la mejorar del clima $(3,92)$ y en el clima de la organización facilita la transferencia $(3,80)$. Sumado a lo anterior, se determina la influencia del apoyo del equipo directivo de la organización $(3,76)$. Los factores débiles en la organización, se vinculan a la baja percepción de una cultura de aprendizaje continuo $(3,46)$, el poco apoyo del equipo de profesionales en la transferencia $(3,46)$ y finalmente, la falta de soporte en los procesos de innovación pedagógica al interior de esta $(3,53)$.

d) Transferencia de competencias. La dimensión valora la percepción de las personas participantes en torno al desarrollo de las prácticas de liderazgo directivo escolar post formación. El grupo de participantes perciben positivamente el desarrollo de competencias, las puntuaciones más altas, la obtienen la gestión directiva, el liderazgo pedagógico, ambas con 4,15 y el trabajo en equipo $(4,11)$, considerándose las competencias con mayor aplicación en la organización, las cuales potencian el desarrollo de la seguridad profesional $(4,07)$. La competencia con menor valoración en la transferencia a la organización, pero sobre la media teórica, es la resolución de conflicto $(3,76)$.

Finalmente, en cuanto a la transferibilidad, el grupo de participantes consideran que la formación es aplicable a todo contexto escolar $(4,26)$. En temas de transferibilidad de las competencias, las mejores puntuaciones son identificadas en el liderazgo pedagógico, la gestión directiva y la gestión curricular, todas con 4,19 puntos. La competencia de resolución de problemas es la de menor valoración con 3,84. Se aprecia una correspondencia entre la transferencia y la transferibilidad de la formación, relacionada a las competencias de liderazgo pedagógico, la gestión directiva y la resolución de problemas. 


\section{3) Facilitadores y obstaculizadores de la transferencia}

En cuanto a los factores facilitadores y obstaculizadores de la transferencia de la formación, se estableció una media de 3,6, estimando un factor facilitador si es $>3,6$ o un factor obstaculizador si es $<3,6$. La justificación de la selección del criterio, se basa en que todas las valoraciones están sobre la media de 3 , ergo, se hizo necesario aumentar la media teórica, para lograr una mejor discriminación de las puntuaciones.

Para la revisión de los facilitadores y obstaculizadores se estableció tres niveles de análisis:

- Primer nivel, se determina la fuerte influencia de los factores personales en el desarrollo personal y en el desarrollo de la seguridad profesional, en este nivel se identifica como factores facilitadores el interés en el tema, la motivación por aprender y la actualización de conocimientos.

- Segundo nivel, se identifica en los factores facilitadores de la formación en el trabajo cooperativo, la reflexión pedagógica, la consecución de objetivos y el clima de aprendizaje. Los factores obstaculizadores se vinculan a la falta de análisis de los nudos de aprendizaje no logrados (feedback) y el limitado uso del feedforward, ambos factores se encuentran asociados al equipo docente durante el proceso formativo.

- Tercer nivel, se aprecian los factores facilitadores de la organización respecto al clima organizacional y el apoyo del equipo directivo. Entre los factores obstaculizadores se distingue la baja percepción de una cultura de aprendizaje continuo, el poco apoyo del equipo de profesionales en la transferencia y la falta de soporte en los procesos de innovación pedagógica al interior del centro escolar.

Para visualizar la clasificación establecida, se diseñó un esquema estructural de los factores facilitadores y obstaculizadores de la transferencia de la formación de competencias de liderazgo directivo escolar, circunscrito a las variables por dimensiones y sus respectivas influencias en las zonas de desarrollo personal, profesional y competencial, como se observa en la Figura 1.

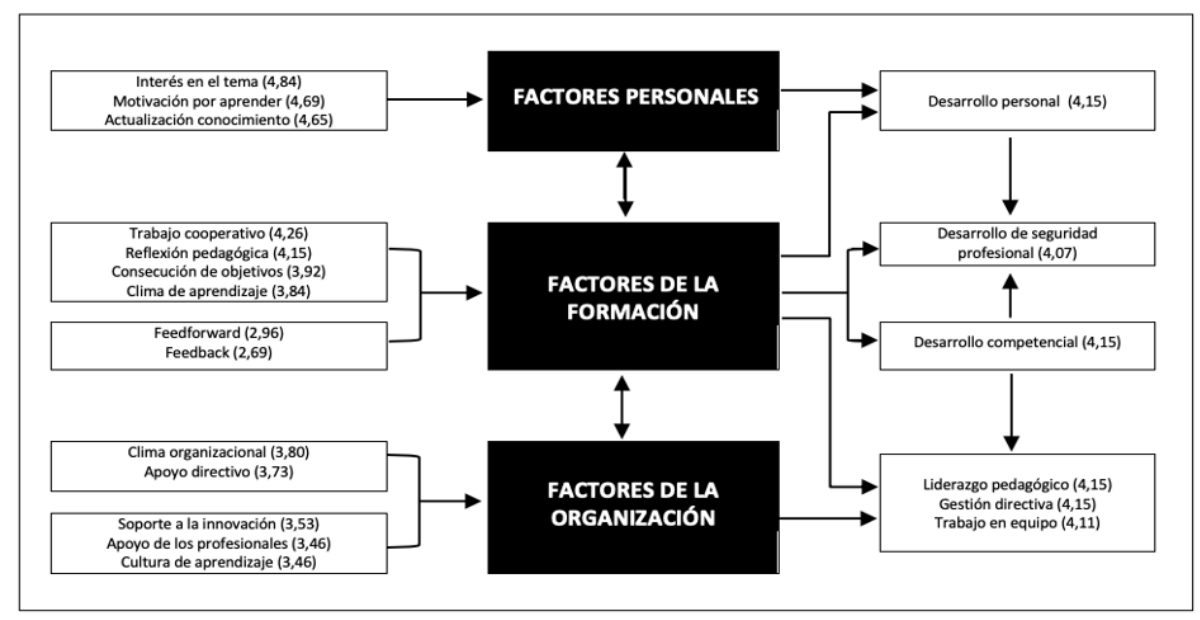

FIGURA 1

Factores facilitadores y obstaculizadores de la transferencia Fuente: Elaboración propia.

A modo de síntesis, los factores de la formación se interrelacionan entre las tres dimensiones. Además, se aprecia que la influencia entre factores es mutua y paralela, las cuales se van interrelacionado para lograr el desarrollo personal y competencial de la persona participante. 


\section{4) Correlaciones entre las dimensiones}

El instrumento de evaluación de la transferencia de competencias de liderazgo escolar, considera cuatro dimensiones: 1) factores personales (FP); 2) factores de la formación (FF); 3) factores de la organización (FO) y 4) transferencia de competencias (TC). Para revisar la existencia de relaciones entre las dimensiones, se decidió aplicar el estadístico de correlación de Pearson (Ver Tabla 3).

TABLA 3

Correlación entre dimensiones

\begin{tabular}{|c|c|c|c|c|c|}
\hline & & $\begin{array}{l}\text { D1. } \\
\text { FP }\end{array}$ & $\begin{array}{l}\text { D2. } \\
\text { FF }\end{array}$ & $\begin{array}{l}\text { D3. } \\
\text { FO }\end{array}$ & $\begin{array}{l}\text { D4. } \\
\text { TC }\end{array}$ \\
\hline $\begin{array}{l}\text { D1. } \\
\text { FP }\end{array}$ & $\begin{array}{l}\text { Correlación de } \\
\text { Pearson Sig. } \\
\text { (bilateral) }\end{array}$ & & & & \\
\hline $\begin{array}{l}\mathrm{D} 2 . \\
\mathrm{FF}\end{array}$ & $\begin{array}{l}\text { Correlación de } \\
\text { Pearson Sig. } \\
\text { (bilateral) }\end{array}$ & $\begin{array}{l}.519^{* * *} \\
.007\end{array}$ & & & \\
\hline $\begin{array}{l}\text { D3. } \\
\text { FO }\end{array}$ & $\begin{array}{l}\text { Correlación de } \\
\text { Pearson Sig. } \\
\text { (bilateral) }\end{array}$ & $\begin{array}{l}.038 \\
.855\end{array}$ & $\begin{array}{l}.406^{*} \\
.040\end{array}$ & & \\
\hline $\begin{array}{l}\mathrm{D} 4 . \\
\mathrm{TC}\end{array}$ & $\begin{array}{l}\text { Correlación de } \\
\text { Pearson Sig. } \\
\text { (bilateral) }\end{array}$ & $\begin{array}{l}215 \\
292\end{array}$ & $\begin{array}{l}.389^{*} \\
.050\end{array}$ & $\begin{array}{l}.489^{*} \\
.011\end{array}$ & \\
\hline
\end{tabular}

Fuente: Elaboración propia

* La correlación es significativa en el nivel 005

${ }^{* *}$ La correlación es significativa en el nivel 0,01

Las dimensiones correlacionadas con significación estadística son:

1. Entre los factores personales y los factores de la formación;

2. Entre los factores de la formación y los factores de la organización y la transferencia de competencia;

3. Entre los factores de la organización y la transferencia de competencias;

4. Entre la transferencia de competencias y los factores de la formación y de la organización.

\section{Discusiones}

Evaluar la formación permite comprender y facilitar la aplicación del programa formativo, por cuanto, identifica los factores facilitadores y obstaculizadores de la transferencia de la formación en la organización escolar, es especial, cuando se trata de una formación continua para el ejercicio del cargo de directora o director de un centro educativo. En este sentido, el estudio cobra relevancia al determinar los factores en los niveles personal (interés por el tema, motivación por aprender y actualización de conocimientos), de la formación (consecución de objetivos, trabajo cooperativo, reflexión pedagógica y el clima de aprendizaje) y de la organización (clima organizacional y apoyo del equipo directivo).

El apoyo del equipo docente durante el proceso formativo (feedback y feedforward) obtuvo las puntuaciones más bajas, por ello, es necesario mejorar los procesos de retroalimentación desde el personal docente en el programa formativo. Por otro lado, los factores obstaculizadores en la organización se vinculan a la baja percepción de una cultura de aprendizaje continuo, la falta de soporte en los procesos de innovación y el poco apoyo del equipo de profesionales en la transferencia, por tanto, es relevante generar las estructuras 
de soporte e incorporar al equipo de profesionales de los centros escolares para facilitar la aplicación de competencias y potenciar una transferencia positiva de la formación.

\section{Conclusiones}

Para entregar coherencia y trazabilidad al artículo, en las discusiones y conclusiones finales, se profundiza en los hallazgos de la investigación, fundamentando y contrastando los resultados obtenidos con las aportaciones de las investigaciones de autoras y autores del marco teórico.

Las conclusiones se desarrollan generando una visión sistémica y una perspectiva integradora del proceso evaluativo de la transferencia de las acciones formativas, considerando: los facilitadores y obstaculizadores; las interrelaciones entre los factores y el desarrollo de competencias de liderazgo escolar. Para tales efectos, el presente apartado se redactó desde la perspectiva de tres grupos de factores condicionantes de la transferencia de la formación: factores personales, factores de la formación y factores de la organización (Baldwin y Ford, 1988).

a) Factores personales. $\mathrm{Al}$ examinar las valoraciones positivas en los factores personales, se concluye la existencia de una fuerte influencia entre los facilitadores de esta dimensión y el desarrollo personal de las personas participantes. Siendo coherente con los aportes de las investigaciones de Cejas y Navío (2017), Feixas, Fernández, Lagos, Quesada y Sabaté (2013), Gessler y Hinrichs (2015) y Rent (2013).

En cuanto a las correlaciones más significativas en los factores personales, se aprecia la correspondencia entre motivación por aprender y el desarrollo personal ( $\mathrm{r}=, 787)$, ambos factores presentan una influencia mutua, a mayor motivación por aprender mayor desarrollo personal. Coincidiendo con los aportes de González et al. (2017) y Gessler y Hinrichs (2015).

b) Factores de la formación. En primer lugar, se determina una valoración positiva del programa de magíster en gestión directiva del plan de formación directoras y directores en la Universidad Mayor. Sumado a lo anterior, se identifica que las personas participantes perciben positivamente el diseño y coherencia interna de la formación, lo cual ofrece mayores posibilidades de transferencia (Feixas et al., 2013; Gessler y Hinrichs, 2015; Kirkpatrick, 2000; López et al., 2017; Rent, 2013).

En el diseño formativo, se aprecia la existencia de una coherencia pedagógica entre la formación, magíster en gestión directiva y el plan de formación nacional. Además, se observa una transversalidad y articulación entre los módulos de aprendizaje centrados en las cuatro dimensiones básicas del liderazgo escolar efectivo, las cuales, son congruentes con las competencias requeridas por el Plan y la política púbica de fortalecimiento de liderazgo escolar (MINEDUC, 2014, Universidad Mayor, 2014).

A pesar de la aseveración anterior, existen obstáculos de la transferencia de la formación, los cuales deben ser considerados a la hora de la toma de decisiones y los ajustes de la acción formativa desde una perspectiva de los bucles de mejora. Desde este enfoque, las limitaciones de formación están focalizadas en los procesos de retroalimentación (feedback) y del feedforward, por parte del equipo docente durante el proceso formativo. Las debilidades mencionadas se alinean con los resultados de la investigación de Feixas et al. (2013) y de Tomàs y Duran (2017).

Los facilitadores se centran en la consecución de los objetivos y en la triada, trabajo cooperativo, la reflexión pedagógica y el clima de aprendizaje. En torno a las estrategias metodológicas, se aprecia una influencia paralela de los factores mencionados con el desarrollo de competencias del liderazgo directivo escolar, entre mayor sea el trabajo cooperativo y la reflexión pedagógica mayor será el grado de desarrollo de competencias. Las conclusiones anteriormente enunciadas, concuerdan con la investigación de Gessler y Hinrichs (2015), convergiendo en la importancia de la práctica reflexiva en el aprendizaje y en los desempeños de las personas participantes, corroborando la mutua influencia entre los factores mencionados. Las correlaciones elaboradas complementan el hallazgo anterior, correspondiéndose altamente, en los siguientes factores: promoción del trabajo cooperativo y promoción de la reflexión pedagógica $(\mathrm{r}=, 718)$; congruencia entre el clima propicio 
para el aprendizaje y la promoción del trabajo cooperativo $(\mathrm{r}=, 645)$; y la interrelación entre el clima y la promoción de la reflexión pedagógica $(\mathrm{r}=, 652)$.

La investigación de Gessler y Hinrichs (2015) identifica la relevancia de la práctica reflexiva en los aprendizajes y desarrollo profesional de las personas participantes. Las estrategias metodológicas de la acción formativa están asociadas a metodologías analíticas deductivas, las cuales resultan adecuadas para el desarrollo competencias. Ambos factores cobran importancia en la formación de equipo directivo, por cuanto, deben ser capaces de liderar procesos pedagógicos, trabajo en equipo centrado en el aprendizaje y a la vez, establecer una dirección en la organización escolar desde una perspectiva de cambio para la mejora escolar.

c) Factores de la organización. En torno a la aplicación de las competencias al puesto de trabajo, las personas participantes consideran que han transferido las competencias de gestión escolar y liderazgo pedagógico al centro escolar durante el proceso de formación, por tanto, se determina una influencia de forma paralela. Existiendo una mutua influencia entre el diseño curricular y la transferencia de competencias a la organización.

Los resultados del análisis de correlaciones, aportan una perspectiva circunscrita a la correspondencia alta desde la mayor seguridad profesional al desarrollo de las competencias de gestión directiva $(\mathrm{r}=, 698)$ y al liderazgo pedagógico $(\mathrm{r}=, 629)$. Entre las correlaciones más significativas, se aprecia la correspondencia positiva entre trabajo en equipo y el desarrollo de la competencia de liderazgo pedagógico $(\mathrm{r}=, 619)$. Por tanto, se concluye que al aumentar la seguridad profesional y fomentar el trabajo en equipo se produce un fortalecimiento en el liderazgo pedagógico.

Las personas participantes perciben como facilitadores de la transferencia son el clima organizacional y el apoyo de los directivos (Baldwin y Ford, 1988; Gessler y Hinrichs, 2015; Holton, 1996; Kirkpatrick, 2000). En los análisis de los resultados se visualiza una mutua influencia entre clima de la organización y el desarrollo de la seguridad profesional, por ende, entre más positivo sea el clima de la organización mayor será la seguridad del profesional para aplicar las competencias en su puesto de trabajo. Dicho hallazgo es ratificado por la correlación alta entre el clima de la organización y la seguridad profesional $(\mathrm{r}=, 717)$.

Los factores obstaculizadores están vinculados a la baja percepción de una cultura de aprendizaje continuo, a la falta de soporte en los procesos de innovación pedagógica y al poco apoyo de los profesionales en la transferencia de la formación a la organización (Baldwin y Ford, 1988; Cejas y Navío, 2017; Gessler y Hinrichs, 2015; González et al., 2017).

A fin de complementar los dos apartados anteriores, se aprecia una correspondencia alta entre el apoyo del equipo directivo y la cultura de aprendizaje continuo $(\mathrm{r}=, 747)$, y una correlación muy alta entre el apoyo del equipo directivo y la innovación pedagógica en la organización $(r=, 874)$. Además, existe una correlación positiva entre el apoyo del equipo de profesionales de la organización y los procesos de innovación pedagógica $(\mathrm{r}=, 709)$, y una correlación muy alta entre el apoyo del equipo de profesionales con la cultura de aprendizaje continuo $(\mathrm{r}=, 859)$. Sumado a alta correspondencia entre la existencia de una cultura de aprendizaje continuo y los procesos de innovación pedagógica $(\mathrm{r}=, 774)$.

La evaluación de la transferencia de la formación es un proceso de levantamiento de información de los cambios producidos por las relaciones causales en niveles personal, profesional y organizacional, atribuibles a un programa formativo. Por tanto, es una herramienta útil para la toma de decisiones, la participación y la rendición de cuentas a la sociedad civil. Desde esta premisa, se considera inherente el potencial de desarrollo de cambio para la mejora presente en la medición de un programa formativo en competencias de liderazgo directivo escolar en el marco del Plan de formación de directoras y directores inserto en la Política de fortalecimiento del liderazgo escolar en Chile. 


\section{Recomendaciones}

En los aspectos internos, es imperante fortalecer el análisis de los nudos deficitarios de las personas participantes (feedback) y motivar el uso del feedforward por parte del equipo docente, dado que ambos factores potencian el proceso de aprendizaje y el desarrollo profesional. El segundo aspecto se identifica en asociar las formaciones a las trayectorias profesionales de las personas postulantes, por cuanto, la experiencia y la carrera profesional son gravitante para lograr una mayor percepción de contribución de las formaciones.

En los aspectos externos, se propone generar redes de seguimiento y acompañamiento de las personas participantes posformación en los diferentes centros educativos, en especial, a los directores y las directoras noveles. Un segundo aspecto, es mantener y fortalecer las redes de profesionales, incorporando al grupo de participantes de las diferentes formaciones con la finalidad de generar redes de contacto y apoyo profesional.

Para ambos puntos, se recomienda diseñar una plataforma web para desarrollar y fortalecer la gestión del conocimiento de las personas participantes de la formación, generando comunidades virtuales, sustentada en un aprendizaje colaborativo y de redes, permitiendo el conocimiento y aprendizaje colectivo, con el propósito de apoyar a las directoras y los directores escolares, en especial, a los y las noveles.

\section{Limitaciones del estudio}

Las limitaciones del estudio se determinan en la ausencia de técnicas y estrategias cualitativas para profundizar en las percepciones del grupo participante en la acción formativa. Además sería oportuno ampliar la muestra de las personas participantes, extendiendo la evaluación de la formación a otras acciones formativas del plan nacional de formación de directoras y directores en Chile.

\section{REFERENCIAS}

Aneas, C., Sánchez, J. y Sánchez, E. (2018). Diseño y validación de dos cuestionarios dirigidos a evaluar la eficacia de la formación del profesorado. Docencia e investigación, 28, 50-76. Recuperado de https://bit.ly/3d7yCee

Baldwin, T. y Ford, J. (1988). Transfer of training: A review and directions for future research. Personnel Psychology, 41, 63-105. doi: http://doi.org/cq3btj

Bernal, C. (2006). Metodología de la investigación. México: Pearson Educación.

Cancino, V. y Vera, L. (2017). Políticas educativas de fortalecimiento del liderazgo directivo: desafíos para Chile en un análisis comparado con países OCDE. Avaliação e Políticas Públicas em Educação, 25(94), 26-58. doi: http ://doi.org/c5nq

Cano, E. (2016). Factores favorecedores y obstaculizadores de la transferencia de la formación del profesorado en educación superior. REICE. Revista Iberoamericana sobre Calidad, Eficacia y Cambio en Educación, 14(2), 133-150. doi: http://doi.org/c2wx

Cebrián, G. (2018). La percepción del desarrollo de competencias directivas en directores de centros educativos en formación. En F. Murillo (Coord.). Avances en democracia y liderazgo distribuido en educación: Actas del II Congreso Internacional de Liderazgo y Mejora de la Educación (pp. 130-135). España: Red de Investigación sobre Liderazgo y Mejora de la Educación. Recuperado de https://bit.ly/2IXnAuf

Cejas, R. y Navío, A. (2017). Formación en TIC del profesorado universitario. Factores que influyen en la transferencia a la función docente. Profesorado. Revista de curriculum y formación de profesorado, 22(3), 271-293. Recuperado de https://bit.ly/2Ug8OEg

Centro de desarrollo del liderazgo educativo [CEDLE]. (2018). Aprendizaje para una política deformación de directores escolares en Chile. Informe CEDLE de politica. Santiago, Chile: Centro de desarrollo del liderazgo educativo. Recuperado de https://bit.ly/3bxLvgI 
Centro de Estudios del Ministerio de Educación [CEM]. (2013). Contextualización de la enseñanza en Chile. Santiago, Chile: Centro de Estudios del Ministerio de Educación. Recuperado de https://bit.ly/2unIOxS

Centro de Perfeccionamiento, Experimentación e Investigación Pedagógica [CPEIP]. (2018). Estadística de los participantes del Plan de formación de directores 2011-2016. Santiago, Chile: Centro de Perfeccionamiento, Experimentación e Investigación Pedagógica.

Costales, Z., Rosales, B. y Acosta, M. (2016). Proyectos educativos y la formación de competencias. Aportes innovadores de la transferibilidad. Revista Iberoamericana de Educación, 71(1), 45-58. doi: http://doi.org/dmb6

Escobar, J. y Cuervo, A. (2008). Validez de contenido y juicio de expertos: una aproximación a su utilización. Avances en Medición, 6, 27-36. Recuperado de https://bit.ly/39mD3iF

Feixas, M., Fernández, A., Lagos, P., Quesada, C. y Sabaté, S. (2013). Factores condicionantes de la transferencia de la formación docente en la universidad: un estudio sobre la transferencia de las competencias docentes. Infancia y Aprendizaje, 36(3), 401-416. doi: http://doi.org/c2wz

García, M. y Caballero, K. (2019). ¿La formación de los equipos directivos es suficiente para desempeñar prácticas eficaces de liderazgo pedagógico? Profesorado. Revista de currículum y formación del profesorado, 23(2), 83-106. doi: http://doi.org/c8xw

Gessler, M. y Hinrichs, C. (2015). Key predictors of learning transfer in continuing vocational training. En S.Bohlinger, U.Haake, C. Helms-Horgensen, H. Toiviainen y A.Wallo. (Eds.), Working and Learning in Times of Uncertainty (pp. 43-60). Roterdam, Países Bajo: Sense Publishers. doi: http://doi.org/dmb8

González, A. Alonso, M. y Berrocal, F. (2017). Evaluación de la eficacia de la formación en la Administración Pública: la transferencia al puesto. Gestión y Análisis de Políticas Públicas, 17, 113-127. doi: http://doi.org/dp3r

González, G., Silvia, I. y Sepúlveda, C. (2016). Desafíos investigativos en la formación inicial de directivos. Revista Iberoamericana de Educación, 70, 43-54. Recuperado de https://bit.ly/2WqeCh9

Hernández, R., Fernández, C. y Baptista, P. (2010). Metodología de la investigación (5a ed.). México: McGraw-Hill.

Holton, E. (1996). The flawed four-level evaluation model. Human Resources Development Quarterly, 7(1), 5-21. doi: http://doi.org/cjv4f3

Kirkpatrick, D. (2000). Evaluación de las acciones formativas: Los cuatro niveles. Barcelona, España: Gestión 2000.

Leithwood, K., Day, C., Sammons, O., Harris, A. y Hopkins, D. (2006). Successful school leadership: What it is and how it influences pupil learning. Research Report 800. United Kingdom: University of Nottingham. Recuperado de https://bit.ly/31Gi7R4

Leithwood, K., Harris, A. y Hopkins, D. (2008). Seven strong claims about successful school leadership. School Leadership and Management, 28(1), 27-42. doi: http://doi.org/bgthwh

López, M., Fijoo, M., Novel, G. y Leyva, J. (2017). Evaluación de la formación continuada. Análisis del proceso de transferencia y sostenibilidad. Atas - Investigação Qualitativa em Saúde, 2, 1165-1174. Recuperado de https:// bit.ly/2Wp1WqE

Ministerio de Educación de Chile [MINEDUC]. (2011). Decreto 44. Reglamentación de la ejecución del Plan de Formación de Directores. Chile: Ministerio de Educación. Recuperado de https://bit.ly/38hbBCE

Ministerio de Educación de Chile [MINEDUC]. (2014). Resolución Exenta N 995. Chile: Ministerio de Educación. Recuperado de https://bit.ly/2tIdQQu

Ministerio de Educación de Chile [MINEDUC]. (2016). Politica de fortalecimiento del liderazgo directivo escolar. Chile: Ministerio de Educación. Recuperado de https://bit.ly/38pi4vi

Muñoz, G. y Marfán, J. (2011). Competencias y formación para un liderazgo escolar efectivo en Chile. Revista de Investigación Educacional Latinoamericana, 48(1), 63-80. doi: http://doi.org/dmb5

Oficina Regional de Educación para América Latina y el Caribe [OREALC/UNESCO]. (2016). Recomendaciones de Politicas Educativas en América Latina en base al TERCE. Santiago, Chile: Oficina Regional de Educación para América Latina y el Caribe. Recuperado de https://bit.ly/3blD9sC

Organización Internacional del Trabajo [OIT]. (2007). Transferibilidad de las calificaciones. Comisión de Empleo y Politica Social. Ginebra, Suiza: Organización Internacional del Trabajo. Recuperado de https://bit.ly/2H5uLj8 
Richar Parra Robledo, et al. Transferencia de la formación en competencias de liderazgo escolar en...

Pont, B., Nusche, D. y Moorman, H. (2008). Mejorar el liderazgo escolar. Volumen 1: Política y Práctica. Paris, Francia: Organización para la Cooperación y el Desarrollo Económicos. Recuperado de https://bit.ly/39mOt66

Rent, A. (2013). La transferencia de aprendizaje en contextos de formación para el trabajo y el empleo. (Tesis doctoral). Universitat Rovira I Virgili, Tarragona, España. Recuperado de https://bit.ly/31FbxtV

Robinson, V., Hohepa, M. y Lloyd, C. (2009). School Leadership and Student Outcomes: Identifying what works and why. Best Evidence Synthesis Iteration [BES]. New Zeland: Ministry of Education. Recuperado de https://bit.l y/38eVWUD

Romero, S., Rodríguez, A. y Gutiérrez, A. (1999). Transferibilidad y orientación para la carrera en la formación profesional. En AIDIPE, IX Congreso Nacional de Modelos de Investigación Educativa. Nuevas realidades educativas, nuevas necesidades metodológicas. Málaga, España. Recuperado de https://bit.ly/31PFrMt

Tomàs, M. y Duran, M. (2017). Comprendiendo los factores que afectan la transferencia de la formación permanente del profesorado. Propuestas de mejora. Revista Electrónica Interuniversitaria de Formación del Profesorado, 20(1), 145-157. doi: http://doi.org/c2w3

Universidad Mayor. (2014). Magister en gestión directiva de excelencia. Programa formativo. Temuco, Chile: Universidad Mayor. Recuperado de https://bit.ly/2H7ZLyT

\section{BY-NC-ND}

